$$
\text { CONF-97/132--2 }
$$

PREPRINT.

Note: This is a preprint of a manuscript being prepared for publication. Contents of this paper should not be quoted or referred to without permission of the author(s).

(submitted to Proceedings of the International Conference on Materials and Process Characterization for VLSI, 1997, Shanghai, China)

\title{
Implantation Processing of Si: A Unified Approach to Understanding Ion-Induced Defects and their Impact
}

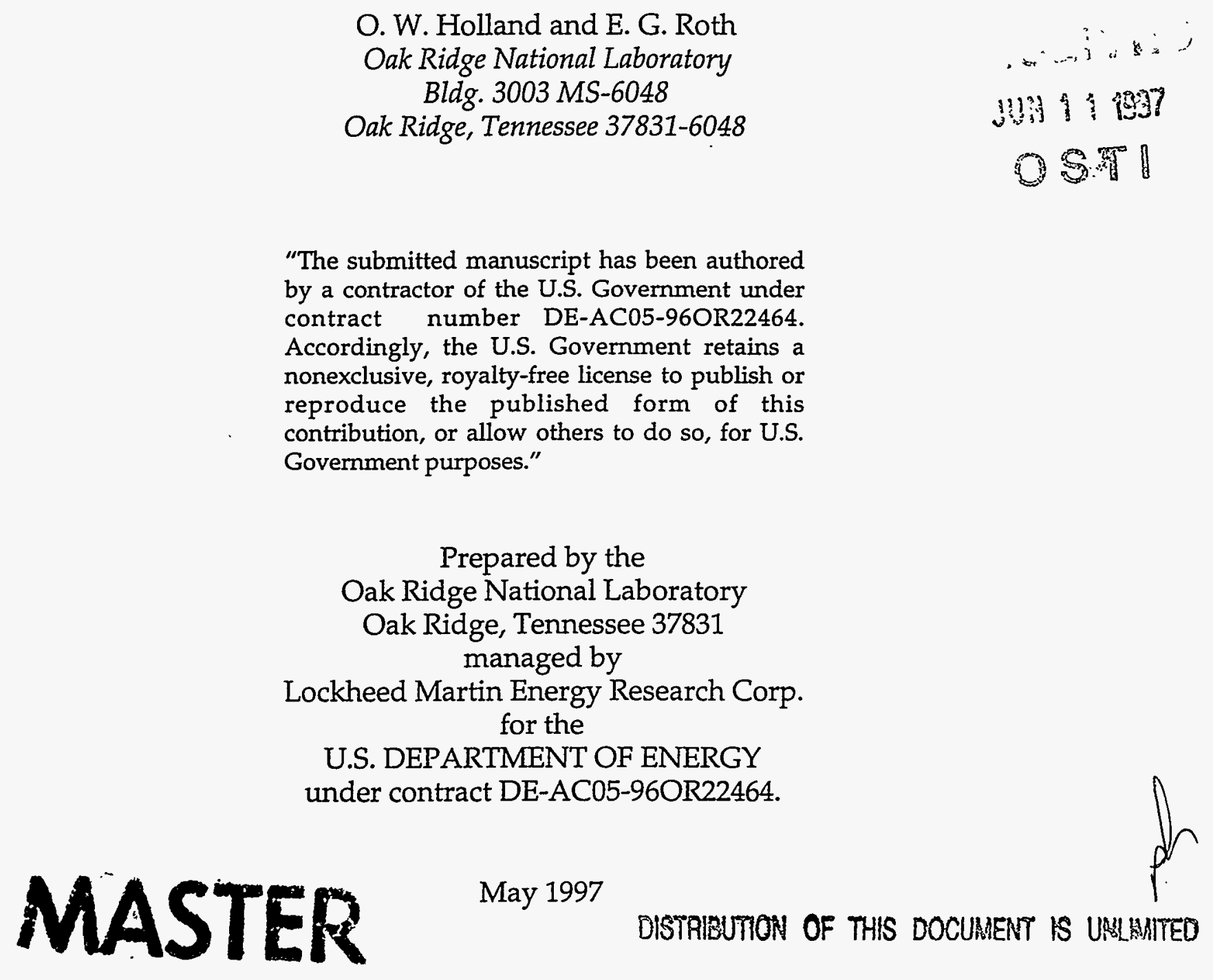




\section{DISCLAIMER}

This report was prepared as an account of work sponsored by an agency of the United States Government. Neither the United States Government nor any agency thereof, nor any of their employees, make any warranty, express or implied, or assumes any legal liability or responsibility for the accuracy, completeness, or usefulness of any information, apparatus, product, or process disclosed, or represents that its use would not infringe privately owned rights. Reference herein to any specific commercial product, process, or service by trade name, trademark, manufacturer, or otherwise does not necessarily constitute or imply its endorsement, recommendation, or favoring by the United States Government or any agency thereof. The views and opinions of authors expressed herein do not necessarily state or reflect those of the United States Government or any agency thereof. 


\section{DISCLAIMIER}

Portions of this document may be illegible in electronic inage products. Images are produced from the best available original document. 


\title{
IMPLANTATION PROCESSING OF Si: A UNIFIED APPROACH TO UNDERSTANDING ION-INDUCED DEFECTS AND THEIR IMPACT
}

\author{
O. W. HOLLAND and E. G. ROTH
}

\begin{abstract}
Oak Ridge National Laboratory, P. O. Box 2008, Oak Ridge, TN 37831-6048
A model is presented to account for the effects of ion-induced defects during implantation processing of $\mathrm{Si}$. It will be shown that processing is quite generally affected by the presence of defect excesses rather than the total number of defects. A defect is considered excess if it represents a surplus locally of one defect type over its compliment. Processing spanning a wide range of implantation conditions will be presented to demonstrate that the majority of the total defects played little or no role in the process. This is a direct result of the ease with which the spatially correlated Frenkel pairs recombine either dynamically or during a post-implantation annealing. Based upon this model, a method will be demonstrated for manipulating or engineering the excess defects to modify their effects. In particular high-energy, selfions are shown to inject vacancies into a boron implanted region resulting in suppression of transient enhanced diffusion of the dopant.
\end{abstract}

\section{INTRODUCTION}

The methodical advance of the semiconductor industry to larger-scale circuit integration is occurring at a rate which cannot be sustained without substantial improvement in manufacturing technology. Aggressive design rules have exposed many weaknesses in existing processes which may require substantial innovation rather than renovation to fix. Some processes may be inherently limited and need to be eliminated, while others suffer from insufficient scientific underpinning to support the needed innovations. Ion implantation is a process used mostly for selective-area doping of $\mathrm{Si}$ and, as such, appears quite frequently in process flows for IC manufacturing. The smaller feature sizes and tolerances mandated by aggressive design rules cause the dopant profiles to be similarly constrained. Historically, electrical junctions formed by implantation were sufficiently deep that they extended well beyond the range of the implanted ions. In such a case, the junction depth is controlled by thermal drive-in and not by the initial implant profile. The situation changed when the design rules mandated junction depths comparable to the range of the implanted ions. Not only does the as-implanted profile now effect the junction depth, but the thermal drive-in is no longer independent of the implantation process. For instance, the presence of ion-induced defects within the dopant distribution causes a transient enhanced diffusion of the dopant during thermal cycling resulting in an unacceptable increase in the junction depth.

This presentation will deal with the scientific basis upon which implantation technology depends. In particular, the impact of defects upon ion beam processing of Si will be explored. A new understanding emerges from this undertaking into the nature of ioninduced defects in Si and their role in various processes. A myriad of ion-related processes will be examined including synthesis, gettering, and doping. These processes span an extended range of ion dose and energy, as well as irradiation temperature. For instance, ion beam synthesis using $\mathrm{O}^{+}$-implantation ${ }^{1,2}$ to form a silicon-on-insulator (SOI) material is nominally performed at elevated temperature $\left(>400^{\circ} \mathrm{C}\right)$ at a dose of $10^{18} \mathrm{~cm}^{-2}$, while doping is done at room temperature (RT) with a dose $\sim 10^{14} \mathrm{~cm}^{-2}$. 
Despite the order-of-magnitude differences in the process parameters, it will be shown that there is a simple model for dealing with ion-induced defects which is common to these processes. In particular, the concept of defect "excesses" will be discussed. An excess exists in the lattice when there is a local surplus of a particular type of defect such as an interstitial over its complimentary defect (i.e. vacancy). Mechanisms for producing such excesses by implantation will be discussed, as well as defect engineering methods to substantially change the nature and distribution of the defect excesses. Not only will the excess defects be shown to play a dominant role in defect-related processing issues, but their manipulation will be demonstrated to be a powerful tool in tailoring the implantation process to achieve design goals.

\section{EXPERIMENT}

A variety of analytical techniques were used in this study including cross-sectional transmission electron microscopy (XTEM) for analysis of lattice damage, and positron annihilation spectrometry for identification of open-volume defects. Lattice strain concomitant to the excess defects was probed by $\mathrm{x}$-ray diffraction techniques. Secondary ion mass spectrometry (SIMS) was used to determine the profile of the implanted dopant. Much of the study concentrated upon bulk Si, either Czochralski or float-zone grown, but a limited use was made of SOI material. The buried $\mathrm{Si}_{\mathrm{SiO}} 2$ interface in the SOI material is an effective, high-temperature barrier to defect fluxes; a condition utilized in experiments designed to develop engineering solutions for problems related to defects.

\section{RESULTS AND DISCUSSION}

Recently, it was shown that during the formation of SOI by separation-by-implantation of oxygen (SIMOX) an excess of vacancy-type defects develops in the superficial Si layer at the surface 3,4 . These defects persist to a critical dose at which point a transition occurs in the damage behavior marked by the appearance of dislocations within this layer. It is of interest to explore conditions similar to those in the SIMOX experiments but using $\mathrm{Si}^{+}$, self-ions. The use of self-ions allows the basic mechanisms of damage formation to be investigated without interference from chemical effects associated with the use of a reactive ion. XTEM micrographs are shown in Fig. 1 from p-type Si(100) implanted at $450^{\circ} \mathrm{C}$ with different fluences of $1.25 \mathrm{MeV} \mathrm{Si}^{+}$-ions. The $2 \times 10^{17} \mathrm{~cm}^{-2}$ implanted sample is seen in Fig. 1(a) to be free of visible defects to a depth of $\sim 1.0 \mu \mathrm{m}$. This occurs despite the fact that the average number of displacements per atom (dpa) within this region is calculated using TRIM ${ }^{5}$ to be $\sim 40$ at this dose. Damage formed near $R_{p}$ is seen to consist of a band of dislocations centered at $\sim 1.5 \mu \mathrm{m}$ which broadens with increasing fluence. The damage morphology at the two higher doses in Figs. 1(b) and 1(c) is seen to be distinctively different than at the lower fluence. A band of dislocation appears within the superficial layer which is spatially isolated by dislocation-free regions on either side. This clearly demonstrates that these dislocations form as a result of defects within the layer rather than at the surface or near $R_{p}$. These results are similar to the those described above for $\mathrm{O}^{+}$-implantation and reported in Refs. (3) and (4). This similarity suggest a common mechanism which was put forth in Ref. (4) to account for the SIMOX results. In this reference, dislocations where shown to form in response to the buildup of lattice strain arising from the accumulation of vacancy-type defects within the layer. 


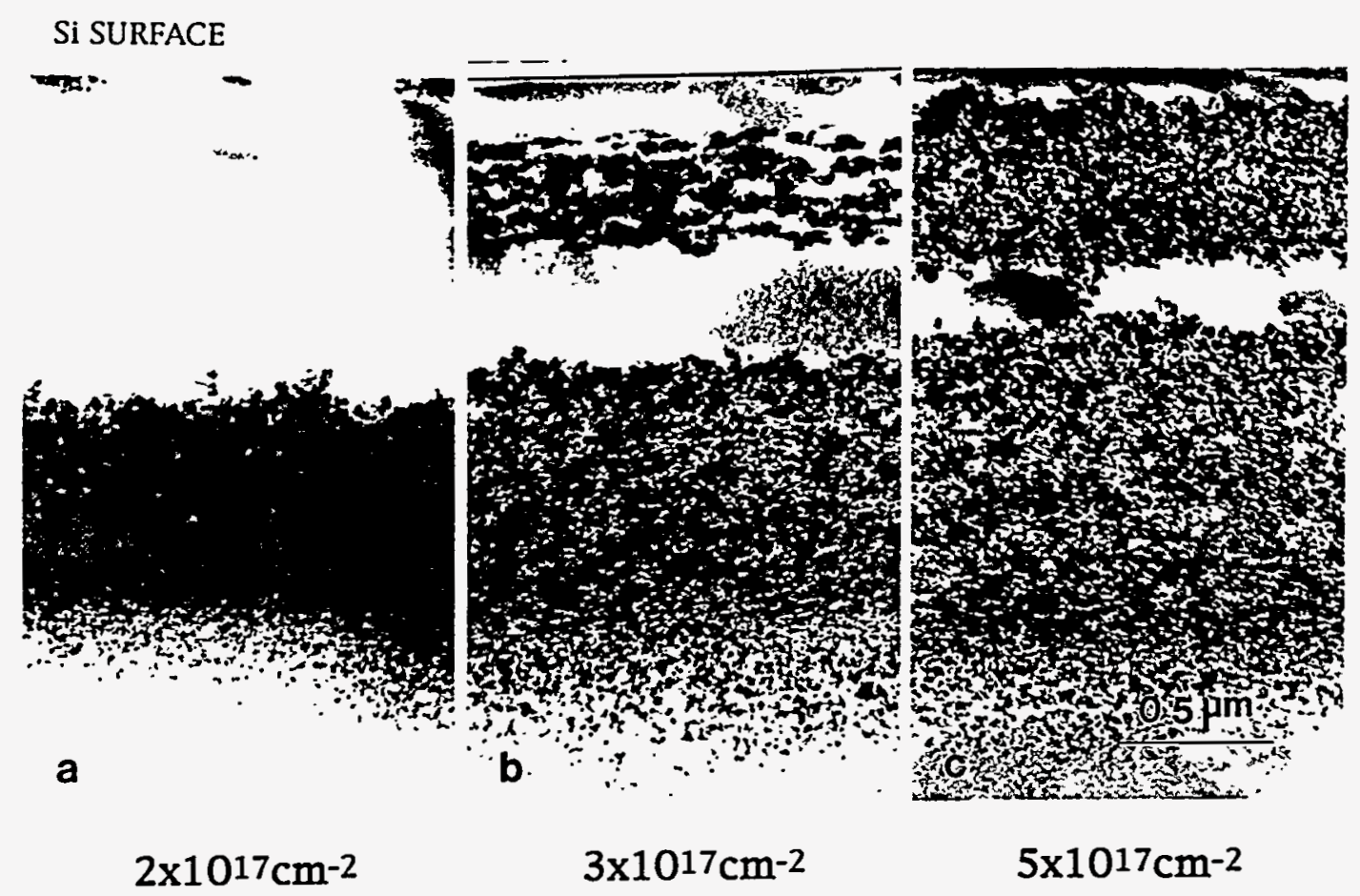

Fig. 1. XTEM micrographs from $\mathrm{Si}(100)$ crystals implanted at $450^{\circ} \mathrm{C}$ with $1.25 \mathrm{MeV}$ self-ions to a fluence of (a) $2 \times 10^{17} \mathrm{~cm}^{-2}$, (b) $3 \times 10^{17} \mathrm{~cm}^{-2}$, and (c) $5 \times 10^{17} \mathrm{~cm}^{-2}$.

Positron annihilation spectroscopy was used to confirm the presence of vacancy -type defects in the $1.25 \mathrm{MeV}$ self-ion implanted samples. The sensitivity of positrons to lattice defects is reflected in a quantity called the S-parameter which measures the Doppler broadening of the $511 \mathrm{keV}$ photon annihilation peak ${ }^{6}$. The S-parameter is calculated by dividing the area of a fixed region in the center of the annihilation peak by the total peak area. Since positrons trapped within an open-volume defect interact less frequently with the higher-momentum, inner-shell electrons, the width of the annihilation spectrum from such defects will be narrower (and the S-parameter larger) than from sites in an undamaged crystal. The dependence of the S-parameter on the incident positron energy (depth) is shown in Fig. 2 for samples implanted with (a) $2 \times 10^{17}$ and (b) $5 \times 10^{17} \mathrm{~cm}^{-2}$; doses below and above, respectively, the critical dose for dislocation growth. At the lower dose, the S-parameter is seen to change rapidly within the superficial layer with a peak value near $0.4 \mu \mathrm{m}$ which is much greater than for bulk $\mathrm{Si}$ $\left(S_{b}=1\right)$. A much smaller $S$-parameter was measured in the sample implanted at the higher fluence of $5 \times 10^{17} \mathrm{~cm}^{-2}$ indicating that dislocation formation in this sample reduces the size or concentration of the vacancy clusters. These results establish an open-volume defect as the precursor to dislocation formation within the superficial layer. A preponderance of these defects, probably small vacancy clusters, accumulate within this region until a critical concentration triggers the transition in the damage behavior. 


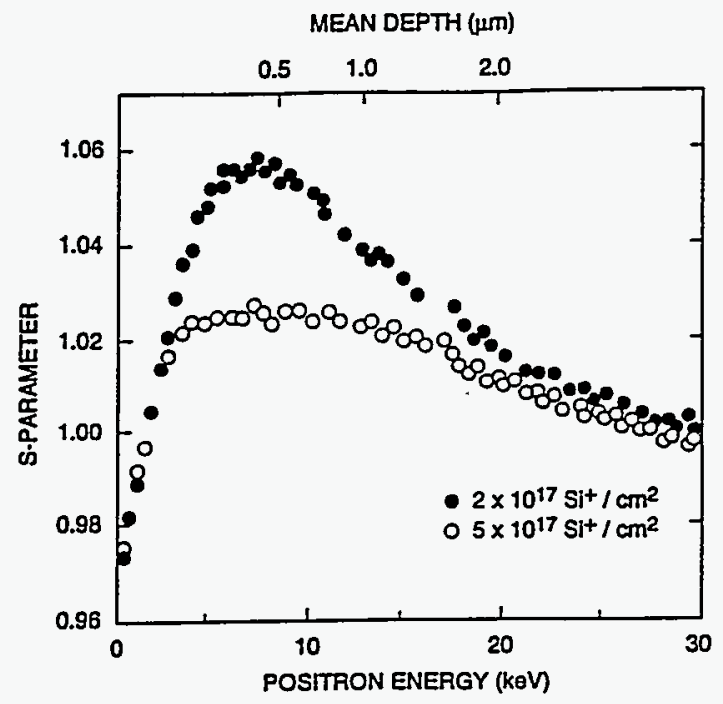

Fig. 2. S-parameter as a function of positron energy in Si(100) implanted at $450^{\circ} \mathrm{C}$ with 1.25 $\mathrm{MeV}$ self-ions. The depth scale at the top of the figure corresponds to the average annihilation depth of the positrons at the energy given by the lower scale.

The self-ion results establish that the accumulation of vacancy-type defects ahead of the ion range is characteristic of the implantation process (albeit under very extreme conditions) and not the ion-solid chemistry. It is possible to account for the preponderance of vacancy-type defects within the superficial layer by a mechanism involving the spatial separation of Frenkel pair defects 7,8 . Interstitial-vacancy pairs are created during irradiation as a result of atomic displacements. The momentum transferred to the interstitial will on average have a non-zero component along the direction of the incident ion. This leads to a spatial separation between the interstitial and vacancy defects with the interstitials distributed at greater depths on average. The difference between the interstitial and vacancy profiles as determined by TRIM are shown in Fig. 3 for $1.25 \mathrm{MeV}$ self-ions normalized to a dose of $2 \times 10^{17} \mathrm{~cm}^{-2}$. It is clear that excess vacancies persists over the top micron layer at a nominal value of 1 at. \%. This value represents only a small fraction (1/4000) of the total vacancies created within this region at this dose. The calculated profile for the vacancy excess matches closely the distribution of vacancy-type defects as indicated in Fig. 1(a) by the vacancy-rich, superficial layer. The identification of this region with the excess vacancy profile presumes that the (spatially) correlated pairs readily recombine so that only the excess defects remain to contribute to damage growth. The elastic continuum approximation can be used to approximate the lattice strain arising from the excess vacancies as calculated from TRIM. These calculations will not be presented, but they do yield values close to the measured value of uniaxial lattice strain of $0.1 \%$ as measured by $\mathrm{x}$-ray diffraction in the $2 \times 10^{17} \mathrm{~cm}^{-2}$ sample. This further establishes the pair separation mechanism as the source of the excess vacancies within the superficial layer.

The discussions to this point have established that spatially correlated Frenkel pairs recombine dynamically during high-temperature irradiation leaving only excess vacancies within an extended region at the surface. These vacancies derive from Frenkel pairs which, on average, become spatially uncorrelated due to the separation mechanism. It is interesting to note that, even in samples implanted at more moderate temperatures, the presence of the excess or net defects can still dominate over the total defect population. 


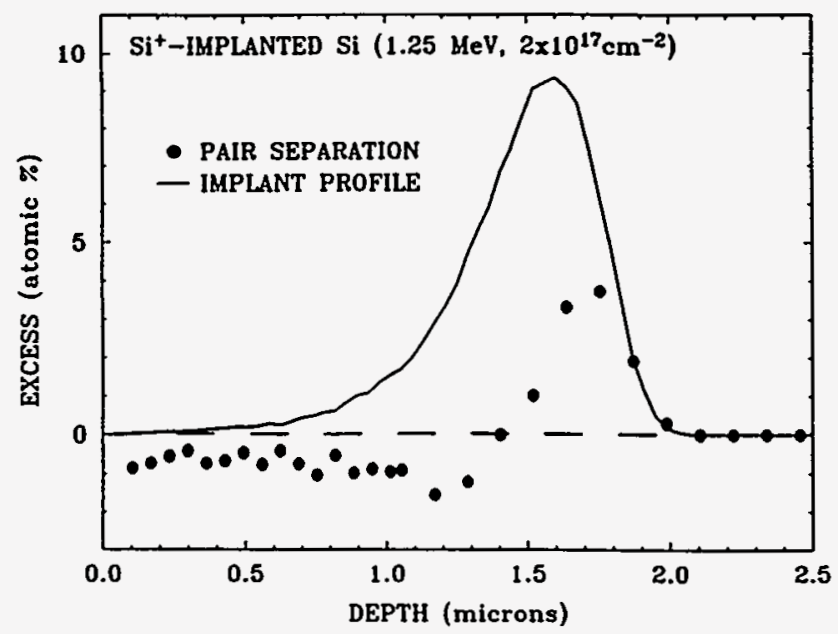

Fig. 3 TRIM calculations for self-ion implantation of $\mathrm{Si}$ at $1.25 \mathrm{MeV}$ normalized to a dose of $2 \times 10^{17} \mathrm{~cm}^{-2}$. Negative values for the defect excess represent a vacancy excess while positive values denote an interstitial excess. The profile of the implanted self-ions is shown for reference.

An example of this can be seen in Fig. 4 which gives data reproduced from Ref. (9) for oxygen gettering at $1 / 2 R_{p}$. This data was accumulated using a variety of different ion species implanted into $\mathrm{Si}$ at different energies and doses. After high-temperature annealing at $1000^{\circ} \mathrm{C} / 60 \mathrm{~min}$., it was observed that gettering of intrinsic oxygen occurred in all samples at a location approximately halfway between the surface and $R_{p}$ (i.e. at $1 / 2 R_{p}$ ). Also shown in the figure is TRIM calculations of the net vacancy concentration at the $1 / 2 R_{p}$ location. It is clear that this calculation accurately reproduces the dependency of the oxygen gettering upon ion mass. This suggests that during annealing the majority of the ion-induced defects are removed except for the excess vacancies which survive to provide sites for trapping the intrinsic oxygen within the region.

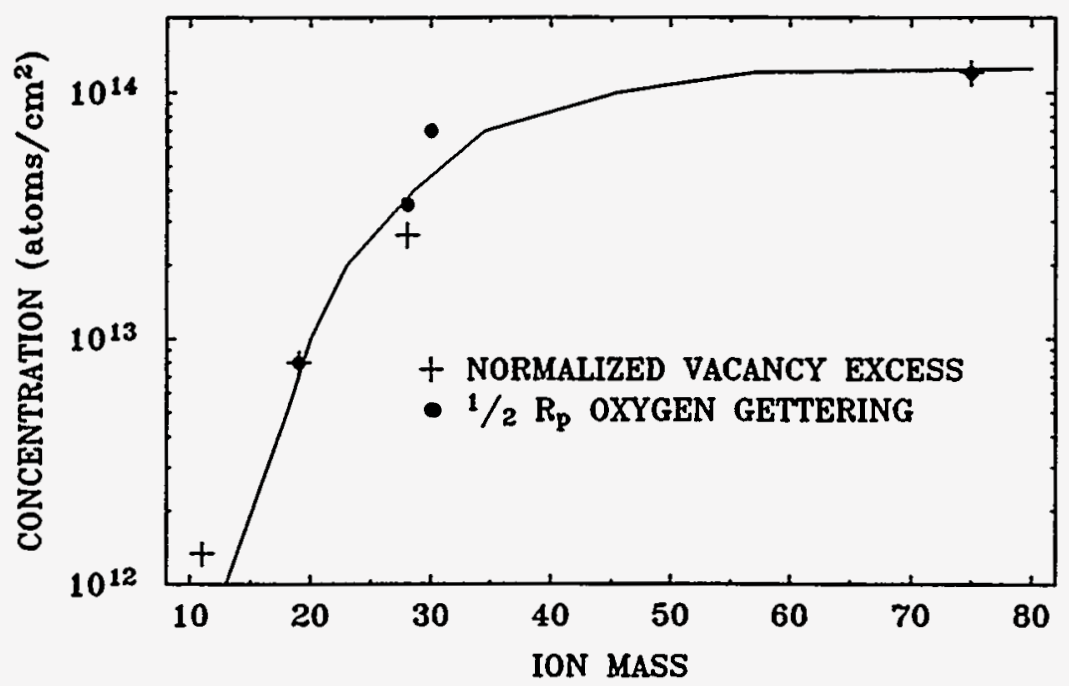

Fig. 4. The ion-mass dependence of the areal concentration of gettered oxygen at $1 / 2 R_{p}$ in implanted $\mathrm{Si}$ as was published in Ref. (9). The excess vacancy concentration at $1 / 2 R_{p}$ was calculated by TRIM simulation for the implantation parameters given in Ref. (9); and subsequently normalized to match the gettered oxygen concentration at mass $=75$. 
The previous examples served to introduce the concept of "excess" defects. Excess vacancies were shown to dominate the damage behavior ahead of $R_{p}$ during elevatedtemperature irradiations. It is clear that there are net or excess interstitials within an extended region centered near $R_{p}$. These arise not only as a result of the pair separation model but also due to the implanted atoms. Since the implanted atoms represent extra atoms within the lattice, they can only be accommodated within the lattice by the generation of interstitials (i.e. one for each implanted atom). This has been referred to as the plus-one model in reference to the effects as observed in $\mathrm{B}^{+}$-implanted Si. Recently, it has been shown that $\{311\}$ defects or interstitial agglomerates are formed during boron implantation near the peak of the implanted profile. 10 The $\{311\}$ 's dissolve during annealing releasing interstitials to participate in the diffusion of the boron which is known to occur by an interstitialcy mechanism. 11 These excess interstitials produce a transient enhanced diffusion (TED) of boron which persists until the interstitials are either eliminated from the diffusion volume or become trapped. The magnitude of the transiency is illustrated in Fig. 5 which compares dopant profiles in $40 \mathrm{keV} \mathrm{B}^{+}$-implanted $\mathrm{Si}$ before and after annealing at $800^{\circ} \mathrm{C} / 15 \mathrm{~min}$. The diffused profile is considerably deeper than the as-implanted one. A finite element analysis as described in Ref. (12) demonstrated that the diffusion was much enhanced over the tracer value (which would yield no movement of the boron). The diffusivity of the boron extracted from this analysis was $6 \times 10^{-14} \mathrm{~cm}^{2} / \mathrm{s}$, which is almost three orders of magnitude greater than the tracer value of $4.3 \times 10^{-17} \mathrm{~cm}^{2} / \mathrm{s}$ at $800^{\circ} \mathrm{C}$. Various methods have been applied including both experimental observation ${ }^{13}$ and modeling ${ }^{14}$ to estimate the numbers of interstitial defects participating in the transiency. Interestingly, the number comes out to be near plus-one (i.e. the implanted dose). Therefore, annealing removes essentially all of the displacement damage except for the plus-one state which survives to produce an enhancement of diffusion.

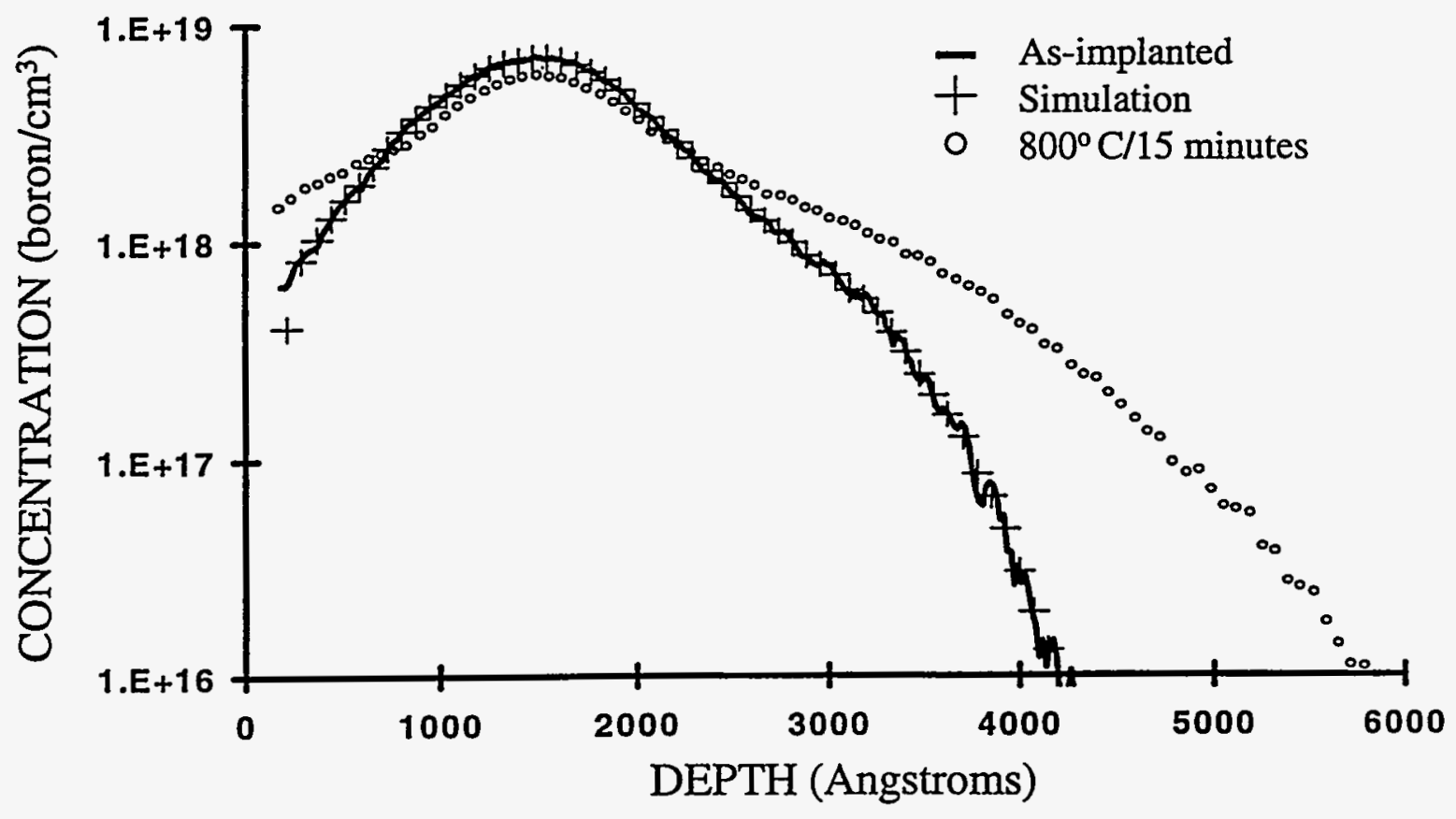

Fig. 5. SIMS profiles of $40 \mathrm{keV}, 10^{14} \mathrm{~B}^{+} / \mathrm{cm}^{2}$ implanted, float-zone $\mathrm{Si}$ at $\mathrm{RT}$. The as-implanted profile is seen to be much shallower than the profile diffused at $800^{\circ} \mathrm{C}$ for $15 \mathrm{~min}$. A finite element analysis $(t)$ shows little or no movement of the boron is anticipated if the diffusivity is given by the boron tracer value. 
The examples presented to this point have a rather remarkable feature in common. Despite large variations in their respective implantation conditions, they to a large degree are dominated by the excess and not total defects. This allows opportunity for engineering the excess defect populations to control their effects. To this end, a method to eliminate TED of boron was devised which involved implanting high-energy self-ions. As indicated earlier, high-energy ions create a vacancy-rich region near the surface and deposit excess interstitials near $R_{p}$. SOI material was chosen for this experiment to isolate the interstitial-type defects formed near $\mathrm{Rp}$ by the self-ions beyond the $\mathrm{Si}_{\mathrm{SiO}}$ interface. It was anticipated that this interface would be a barrier to back diffusion of the interstitials, in essence, preventing any recombination between the excess vacancies and interstitials over an extended temperature range. In this way, the high-energy ions are used to selectively inject vacancies into the top Si layer. Therefore, coimplantation of self-ions and boron at energies which effectively overlap the boron plus-one interstitials with the vacancy-rich region created by the self-ions creates a situation where the defects can mutually annihilate each other. The coimplanted SOI samples were implanted with $40 \mathrm{keV} \mathrm{B}$--ions at a dose of $10^{14} \mathrm{~cm}^{-2}$ along with $2.0 \mathrm{MeV}$ self-ions at a dose of $5 \times 10^{15} \mathrm{~cm}^{-2}$. The boron implant resulted in a peak dopant concentration of $10^{19} \mathrm{~cm}^{-2}$ while the self-ions produced a net vacancy concentration within the top Si layer of about $5 \times 10^{18} \mathrm{~cm}^{-2}$ (as determined by TRIM). Fig. 6 gives a comparison of the dopant profiles under various conditions. It is clear that the diffused profiles (after $800^{\circ} \mathrm{C}$ annealing) demonstrate that the motion of the boron within the coimplanted sample has been substantially retarded over the control (e.g. a $\mathrm{B}^{+}$-only implanted sample). While there is some anomalous outdiffusion of the boron in the coimplanted sample, the technique seems to be very effective in limiting the diffusion of boron and thus, the depth of the electrical junction.

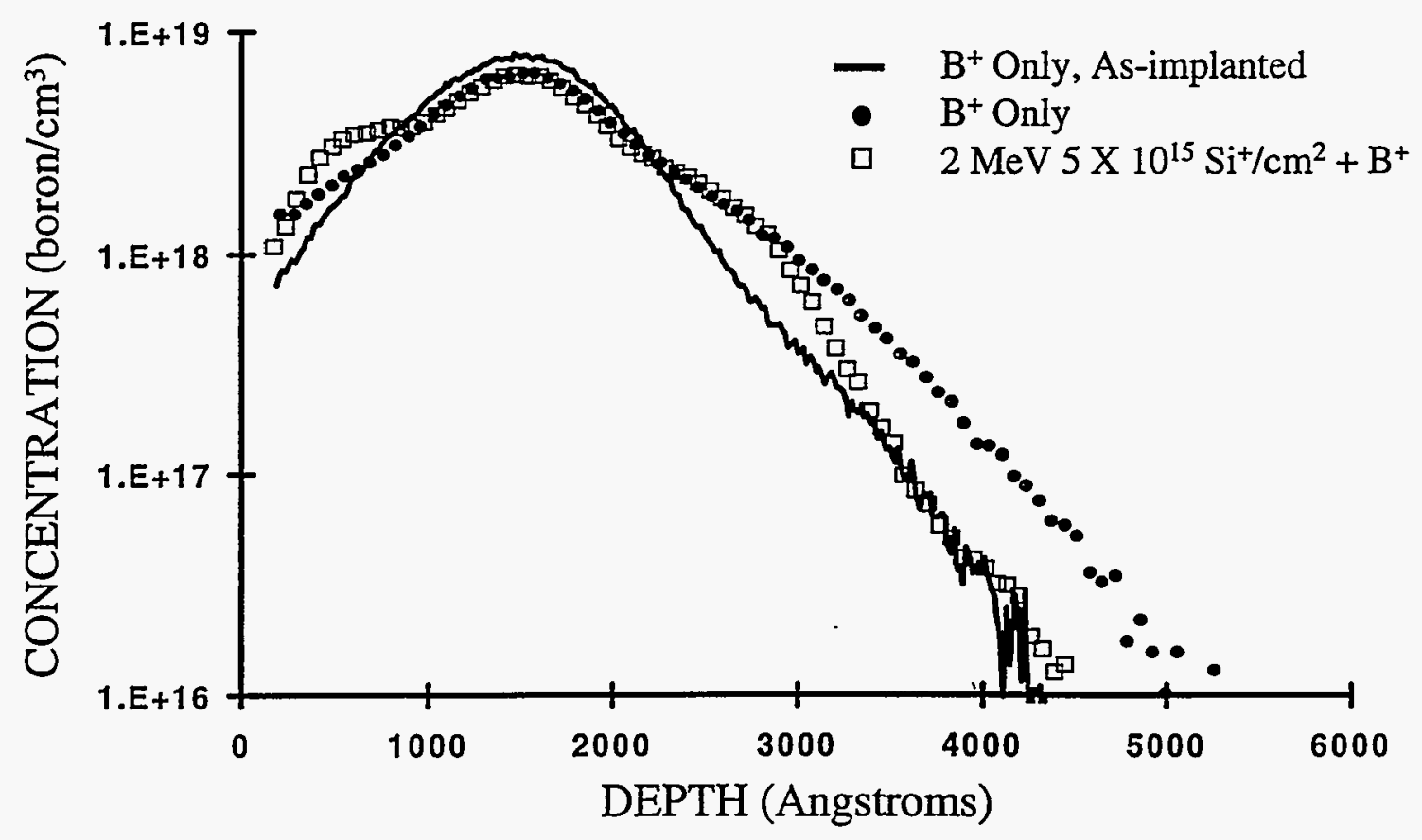

Fig. 6. Comparison of SIMS profiles of $40 \mathrm{keV}, 10^{14} \mathrm{~B}^{+} / \mathrm{cm}^{2}$ implanted SOI after annealing at $800^{\circ} \mathrm{C} / 15 \mathrm{~min}$. The sample coimplanted with $2.0 \mathrm{MeV}$ self-ions at a dose of $5 \times 10^{15} \mathrm{~cm}^{-2}$ is seen to exhibit much less indiffusion of the boron than in the sample implanted with $\mathrm{B}^{+}$-only. The as-implanted boron profile is shown for reference. 


\section{CONCLUSIONS}

A model was presented to account for the effects of ion-induced defects during implantation processing of Si which depended only upon the presence of defect excesses. It was demonstrated that under a wide range of implantation conditions, the majority of the total defects played little or no role in the process. This is a direct result of the ease with which the spatially correlated Frenkel pairs can recombine, either dynamically or during a post-implantation anneal. Based upon this model, a method was demonstrated for manipulating or engineering the excess defects to modify their effects. In particular high-energy, self-ions were used to inject vacancies into a boron implanted region. These vacancies acted as an artificial recombination tool to eliminate the plus-one interstitials and thus suppress the transient enhanced diffusion of boron.

\section{ACKNOWLEDGMENT}

The contribution of $\mathrm{B}$. Nielsen of Brookhaven National Laboratory in providing the positron annihilation results is acknowledged. Research was sponsored by the Division of Materials Science, U.S. Department of Energy under Contract DE-AC05-960R22464 with Martin Marietta Energy Systems, Inc.; and by Oak Ridge Associated Universities under contract DE-AC05-76OR00033 with the U. S. Department of Energy.

\section{REFERENCES}

[1] H. H. Hosack, T. W. Houston, and G. P. Pollack, Sol. State Tech. 12, 61 (1990).

[2] M. A. Guerra, Sol. State Tech. 11, 75 (1990).

[3] O. W. Holland, D. S. Zhou, and D. K. Thomas, Appl. Phys. Lett. 63, 896 (1993).

[4] D. S. Zhou, O. W. Holland, and J. D. Budai, Appl. Phys. Lett. 63, 3580 (1993).

[5] J. Biersack and L. Haggmark, Nucl. Instr. and Meth. 174, 257 (1980).

[6] Peter J. Schultz and K. G. Lynn, Review of Modern Physics, 60, 701 (1988).

[7] A. M. Mazzone, Phys. Status Solids A 95, 149 (1986).

[8] O. W. Holland and C. W. White, Nucl. Instr. and Meth. B59/60, 353 (1991).

[9] M. Tamura, T. Ando and K. Ohyu, Proceedings of the Seventh International Conference on Ion Beam Modification of Materials, edited by S. P. Withrow and D. B. Poker (NorthHolland, Amsterdam, 1991), p. 572.

[10] D. J. Eaglesham, P. A. Stolk, H. -J. Gossman, and J. M. Poate, Appl. Phys. Lett. 65, 2305 (1994).

[11] P. M. Fahey, P. B. Griffin, and J. D. Plummer, Rev. Mod. Phys. vol. 61, no. 2, 289 (1989).

[12] O. W. Holland and J. Narayan, Nucl. Instr. and Meth. B40/41, 537 (1989).

[13] D. J. Eaglesham, P. A. Stolk, H. -J, Gossman, T. E. Haynes and J. M. Poate, Nucl. Instr.and Meth. B106, 191 (1995).

[14] M. D. Giles, J. Electrochem. Soc. 138, 1160 (1991). 